\title{
Low Concentration Epidural Anesthesia Combined with Transversus Abdominis Plane Block -- A Case Report in A High-risk Colostomy Operation
}

\author{
Tzu-Chun Wang ${ }^{1, \star}$, Chia-Hsiang Huang ${ }^{1}$
}

\author{
${ }^{1}$ Department of Anesthesiology, Taitung \\ Mackay memorial hospital, Taitung, \\ Taiwan \\ *Correspondence \\ ff_west@hotmail.com \\ (Tzu-Chun Wang)
}

\begin{abstract}
We report a high-risk cachexia patient receiving special regional anesthesia in a colostomy operation. Because of multiple underlying diseases and severe malnutrition status, we combined epidural anesthesia (EA) with transversus abdominis plane (TAP) block. Low concentration EA (Lidocaine 1\% as test dose and rescue dose; Bupivacaine $0.2 \%$ as loading and maintenance dose) aimed for visceral pain; subcostal TAP block (Bupivacaine $0.25 \%$ ) focused on the skin incision. The colostomy was done completely without any complication. Our case showed that low concentration EA combined with TAP block provided stable and sufficient anesthesia and could be a sole anesthesia technique in a colostomy.
\end{abstract}

\section{Keywords}

Epidural anesthesia, Truncal blocks, Transversus abdominis plane block, Critical Care

\section{Introduction}

Previous studies have largely focused on the comparison between epidural anesthesia (EA) and transversus abdominis plane (TAP) block, while little is known about combining of them.

EA is broadly used in many anesthesia and analgesia fields, and known for its complications such as hypotension and hemodynamic instability.[1] In contrast, TAP block has shown many benefits in abdominal analgesia over the past decade.[2] Studies have revealed an equal analgesia effect between TAP block and EA in abdominal surgery,[3-5] esophagectomy, [6] laparoscopic colorectal surgery [7], and transperitoneal laparoscopic nephrectomy.[8] Furthermore, TAP has lower complications than EA such as hospital length of stay, hypotension and the need for volume resuscitation. [3, 4, 6] However, TAP block has little analgesic effect for visceral pain, and could hardly be a major anesthesia technique in major abdomen surgeries.[9] Some reports also point out advantages of EA, including the quality of analgesia[10,11] and opioid consumption reducing.[11]

In order to optimize the analgesic effect and reduce complications, several attempts have been made to combine EA with TAP block as an adjunctive analgesia technique.[12, 13] However, the optimal dose, concentration, and volume in this combination were still lacking in previous literature. Moreover, none of them was used as a major anesthetic technique perioperatively. We herein present an alternative anesthetic choice for a high-risk cachexia patient: low concentration epidural anesthesia combined with transversus abdominis plane block.

Institutional Review Board approval has been obtained for this study.

\section{Case report}

A man in his seventies had the past history of hypertension, intracranial hemorrhage, and rectal cancer in stage cT3N2bM0 with palliative care. He was sent to our hospital owing to malignant rectal obstruction. Besides, cachexia with severe malnutrition, tumor bleeding and anemia ( $\mathrm{Hb} 5.8 \mathrm{~g} / \mathrm{dL}$ ), relatively hypotension status (systolic blood pressure 80 - $90 \mathrm{mmHg}$ ), and grade II pressure sore over the hip area were also noted. Therefore, Our ICU team gave adequate intravenous fluid and nutrition support, blood transfusion for anemia and bleeding tendency, prophylaxis antibiotics for the intra-abdominal in-

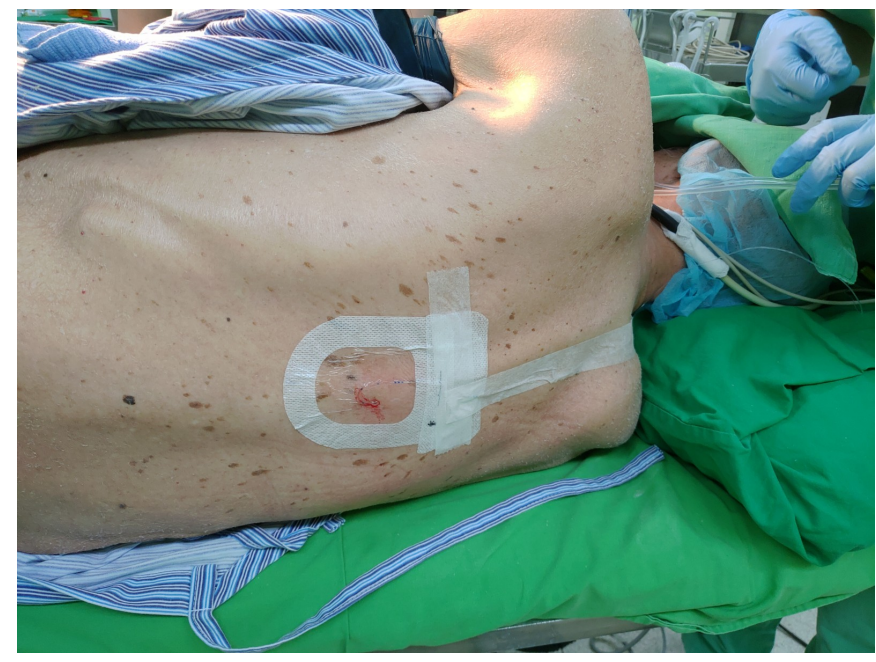

F I G URE 1. Location of epirudal catheter between thoracic vertebrate 8 th and 9 th. 


\section{A}

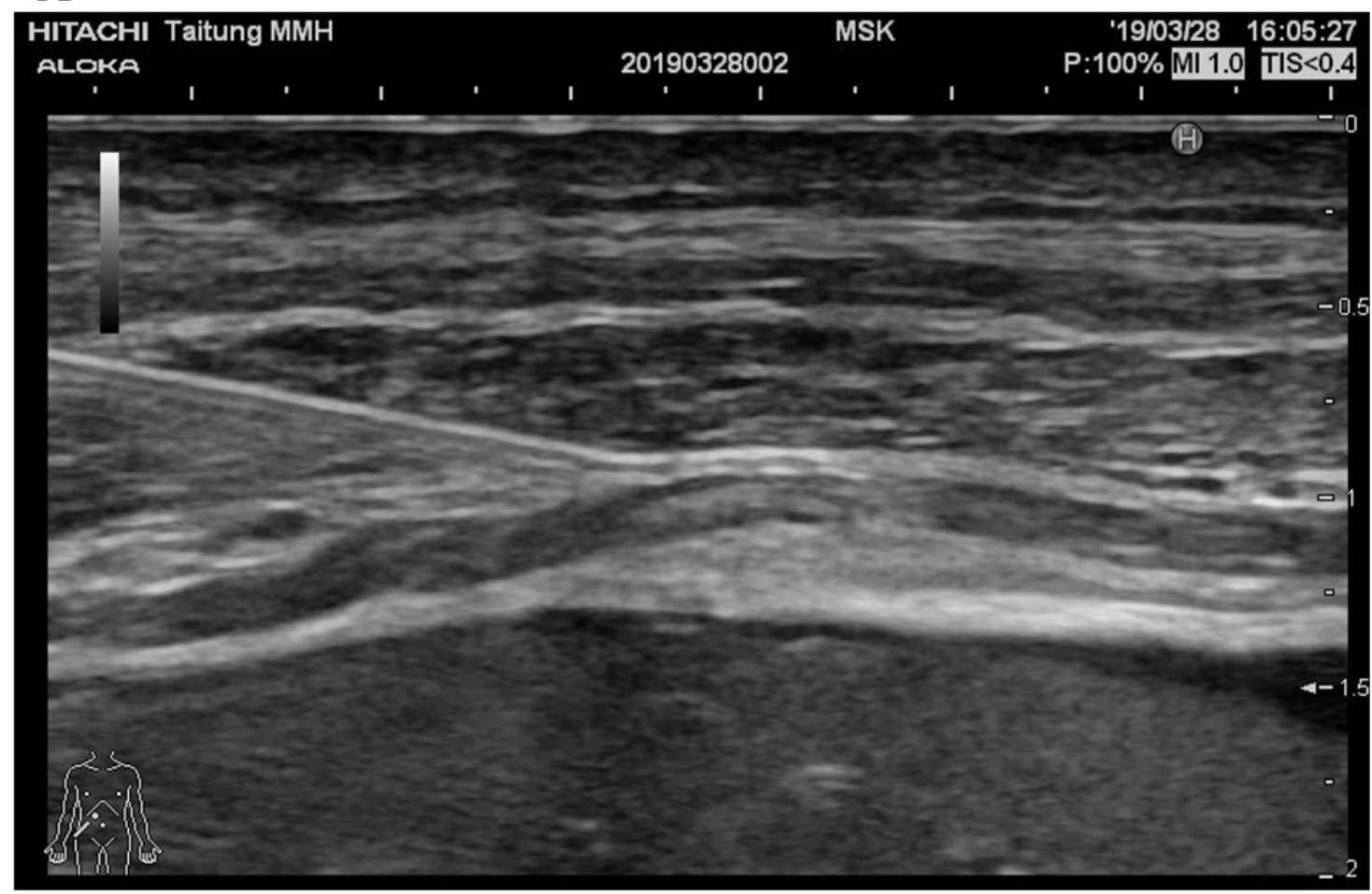

$\mathrm{B}$

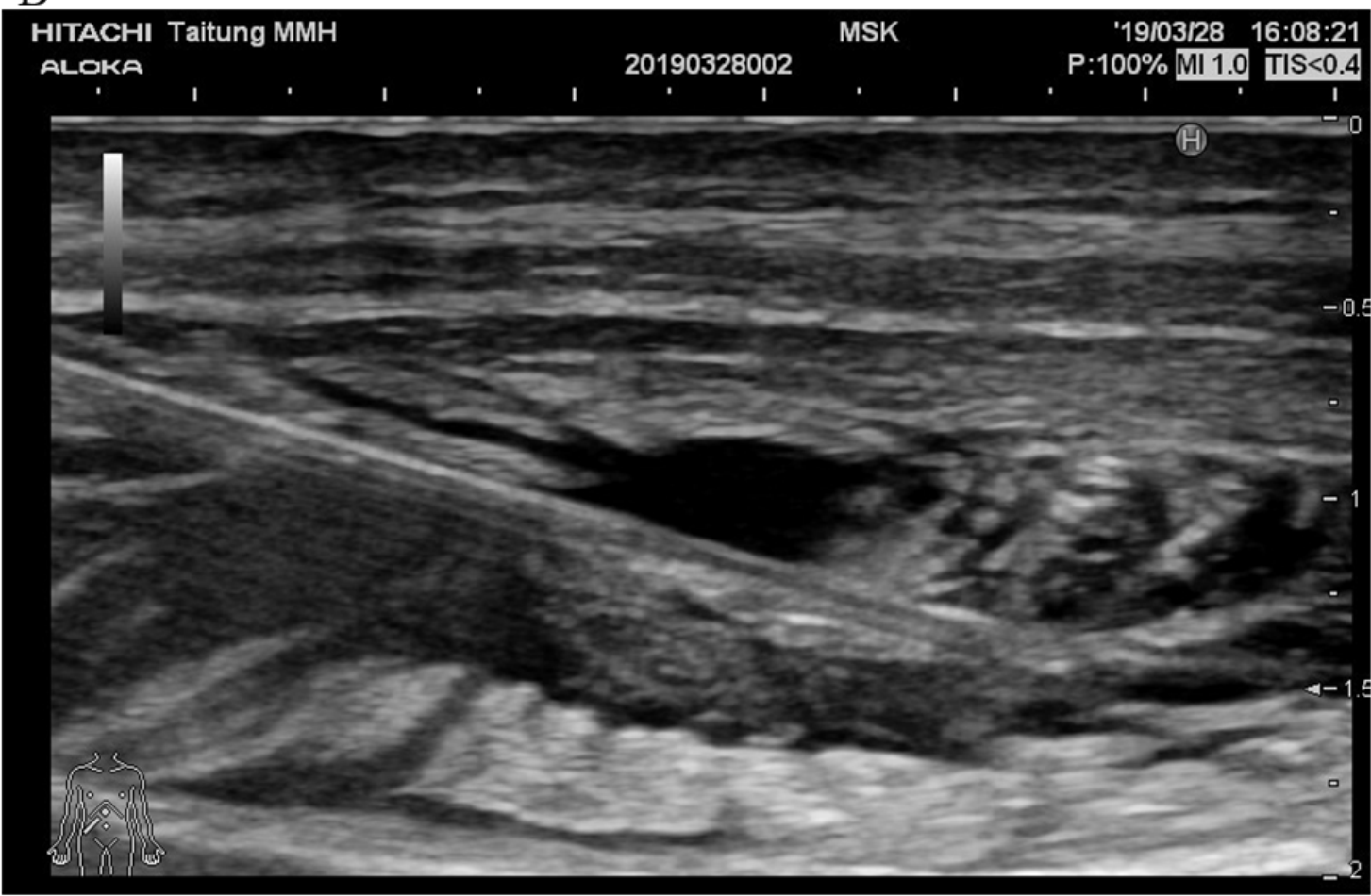

F I G U R E 2. Sono-guide subcostal transversus abdominis plane block with $20 \mathrm{ml}$ of Bupivacaine $0.25 \%$.

fection, and wound care for the pressure sore. After 1 week of ICU admission, his hemodynamic status gradually improved (systolic pressure over $90-100 \mathrm{mmHg}$ ). His electrolyte imbalance and anemia were also corrected ( $\mathrm{Hb} 7-8 \mathrm{~g} / \mathrm{dL})$. However, he still suffered from abdominal distension pain, poor appetite, and intermittent fever because of tumor-related rectal obstruction. To prevent further complications of malignant obstruction, our team had to releaase his rectal obstruction. Due to the size and location of his rectal tumor, supportive care or colonic stenting might have a low success rate. But the risks in general anesthesia including complications of intubation, delayed extubation, and tracheostomy frustrated this patient and his family to receive colostomy operation. On the other hand, traditional neuraxial anesthesia may accompany with hemodynamic unstable, especially in this chronic malnutrition patient. To avoid those complications, we discussed with our surgeon and patient the operation area, anesthetic choices and risks. Finally, we decided lower dose epidural anesthesia 


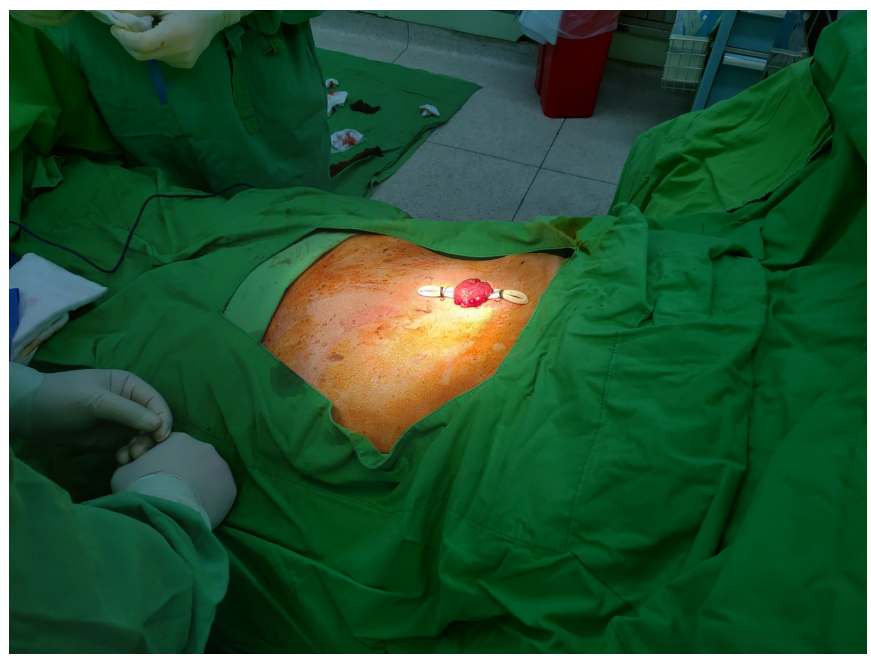

FIGURE 3. Colostomy in right upper quadrate abdomen.

which was aimed for visceral pain, combined with subcostal TAP block for skin incision.

Under standard monitoring, we injected subcutaneous local anesthesia ( $2 \mathrm{ml}$ of Lidocaine $0.1 \%$ ) and then indwelled epidural catheter to space T8 - T9 with depth $4.5+5.5 \mathrm{~cm}$ (Fig. 1). Test dose $3 \mathrm{ml}$ of Lidocaine 1\% with adrenaline 1:400,000 was given then. After 5 minutes of uneventful observation, we gave a loading dose $5 \mathrm{ml}$ of Bupivacaine $0.2 \%$ and $50 \mathrm{mcg}$ of Fentanyl via the epidural catheter. The anesthetic effect was tested successfully by skin sensory testing to both cold and touch stimuli. Moreover, there was no undesirable hemodynamic change after the loading dose. Next, right side subcostal TAP block with $20 \mathrm{ml}$ of Bupivacaine $0.25 \%$ was done under sono-guide smoothly (Fig. 2). The vital sign was stable before and after EA and TAP block (Before: BP 123/48 mmHg, HR 80/min; After: BP 130/38 mmHg, HR $80 / \mathrm{min}$ ). Besides, we also in advance prepared Lidocaine $1 \%$ with adrenaline $1: 400,000$ as a rescue dose via epidural, Bupivacaine $0.2 \%$ with Fentanyl as maintenance dose via epidural.

After 40 minutes of preparation, the surgeon started colostomy. This patient was comfortable in the beginning. No supplementary or rescue dose was needed during abdominal incision. Moreover, our surgeon was satisfied with the muscle tone and operation field under EA with TAP block. However, after 10 minutes of operation, the patient felt mild abdominal tightness while colon traction. Therefore, rescue dose $2 \mathrm{ml}$ of Lidocaine 1\% with adrenaline 1:400,000 via epidural was given immediately, and small dose intravenous Fentanyl $50 \mathrm{mcg}$ was added for augmentation of analgesia and sedative effect. This traction discomfort was relieved less than 3 minutes, and colostomy proceeded without any tightness sensation. In the middle of the operation, $3 \mathrm{ml}$ of Bupivacaine $0.2 \%$ with $25 \mathrm{mcg}$ of Fentanyl was given as maintenance dose. The patient remained clear consciousness and stable hemodynamic status during the whole operation. Finally, colostomy was smoothly finished for an hour (Fig. 3), and post-operative ICU supportive care was arranged. This patient remained stable vital sign and started trying diet less than 24 hours after this operation.

\section{Discussion}

Anesthesia combined lower dose EA with TAP block in our high-risk patient was stable and successful. It showed the potential benefit in combining different regions and doses in regional anesthesia.

Recommended doses of EA have been used for a long time, such as $2 \%$ Lidocaine, $0.5 \%-0.75 \%$ Bupivacaine, and 0.75 - $1.0 \%$ Ropivacaine [1]. Under these dosages, considerable complications including hypotension occurred in critical patients. To prevent these undesirable events, some anesthesiologists have special techniques including decreasing EA volume, combining EA with multimodal analgesia, or combining EA with general anesthesia. For example, combining low volume EA with peripheral nerve block was successfully applied in the postoperative analgesia in cesarean section [2]; Combining EA with TAP as an adjuvant analgesic tool in general anesthesia was helpful in carina tumor resection [3].

However, low concentration EA with peripheral block has never been examined in previous reports. In this case, we carefully titrated EA with low concentration local anesthetic agent, including Lidocaine $1 \%$ (half of the traditional concentration) and Bupivacaine $0.2 \%$ (less than half of the traditional concentration) with little opioid, to block intestinal and visceral pain; Meanwhile, to achieved adequate anesthetic effect for skin incision, we applied subcostal TAP block on right upper quadric abdomen. The total regional anesthetic dose was less than the systemic toxic dose (50mg of lidocaine, $16 \mathrm{mg}$ of bupivacaine and $75 \mathrm{mcg}$ of Fentanyl in epidural space, $50 \mathrm{mcg}$ of bupivacaine in TAP block). During the whole operation, we only used a light additive intravenous drug (50mcg of Fentanyl). This combination had sufficient anesthesia effect and kept this patient in stable hemodynamic status.

Although this conception could be widely used in critical patients, the minimal effective dose for this combining is still a question. In our case, incision pain of abdominal skin and muscle was effectively blocked by the subcostal TAP block (20ml of Bupivacaine $0.25 \%$ ). But for visceral pain, especially while colon traction, our initial doses ( $3 \mathrm{ml}$ of Lidocaine $1 \%$ with adrenaline $1: 400,000 \& 5 \mathrm{ml}$ of Bupivacaine $0.2 \%$ with $50 \mathrm{mcg}$ of Fentanyl) seems not enough for colon traction. It may be caused by two different reasons. First, to block colon sensation, less than half of traditional concentration in EA may be insufficient. Our case had breakthrough pain with Bupivacaine $0.2 \%$ with additive opioid (50mcg of Fentanyl), but this pain was relieved after rescue dose $2 \mathrm{ml}$ of Lidocaine $1 \%$. Second, to cover the visceral pain of colostomy thoroughly, we may need a larger volume (more than $10 \mathrm{ml}$ ) to cover their complex neural distribution of colon. Because of different neural distribution and sensitivity in various operations, we need further discussion about the best concentration and volume for other abdominal and thoracic surgeries. To reduce complications and to improve the quality of regional anesthesia, we hope there will be more temptation in the combination of regional anesthesia. 


\section{ACKNOWLEDGMENTS}

The authors have no sources of funding to declare for this manuscript.

\section{CONFLICTS OF INTEREST}

The authors declare no conflicts of interest regarding the publication of this article.

\section{GLOSSARY OF TERMS:}

EA: epidural anesthesia.

TAP: transversus abdominis plane.

\section{REFERENCES}

[1] Miller RD. Miller's anesthesia. Philadelphia, PA; Elsevier/Saunders; 2015. p. 2 volumes (3270, I-3122 pages).

[2] Tsai HC, Yoshida T, Chuang TY, et al. Transversus Abdominis Plane Block: An Updated Review of Anatomy and Techniques. Biomed Res Int. 2017;2017:8284363.

[3] Shaker TM, Carroll JT, Chung MH, et al. Efficacy and safety of transversus abdominis plane blocks versus thoracic epidural anesthesia in patients undergoing major abdominal oncologic resections: A prospective, randomized controlled trial. Am J Surg. 2018;215:498-501.

[4] Baeriswyl M, Zeiter F, Piubellini D, et al. The analgesic efficacy of transverse abdominis plane block versus epidural analgesia: A systematic review with meta-analysis. Medicine (Baltimore). 2018;97:e11261.

[5] Ganapathy S, Sondekoppam RV, Terlecki M, et al. Comparison of efficacy and safety of lateral-to-medial continuous transversus abdominis plane block with thoracic epidural analgesia in patients undergoing abdominal surgery: A randomised, open-label feasibility study. Eur J Anesthesiol. 2015;32:797-804

[6] Levy G, Cordes MA, Farivar AS, et al. Transversus Abdominis Plane
Block Improves Perioperative Outcome After Esophagectomy Versus Epidural. Ann Thorac Surg. 2018;105:406-412.

[7] Niraj G, Kelkar A, Hart E, et al. Comparison of analgesic efficacy of four-quadrant transversus abdominis plane (TAP) block and continuous posterior TAP analgesia with epidural analgesia in patients undergoing laparoscopic colorectal surgery: an open-label, randomised, noninferiority trial. Anesthesia. 2014;69:348-355.

[8] Aditianingsih D, Mochtar CA, Chandra S, et al. Comparison of ThreeQuadrant Transversus Abdominis Plane Block and Continuous Epidural Block for Postoperative Analgesia After Transperitoneal Laparoscopic Nephrectomy. Anesth Pain Med. 2018;8:e80024.

[9] Connolly NC. Real-world insights on the use of transversus abdominis plane block with liposomal bupivacaine in the multimodal management of somatic versus visceral pain in the colorectal surgery setting. J Pain Res. 2018;11:1141-1146.

[10] Canakci E, Gultekin A, Cebeci Z, et al. The Analgesic Efficacy of Transverse Abdominis Plane Block versus Epidural Block after Caesarean Delivery: Which One Is Effective? TAP Block? Epidural Block? Pain Res Manag. 2018;2018:3562701.

[11] Iyer SS, Bavishi H, Mohan CV, et al. Comparison of Epidural Analgesia with Transversus Abdominis Plane Analgesia for Postoperative Pain Relief in Patients Undergoing Lower Abdominal Surgery: A Prospective Randomized Study. Anesth Essays Res. 2017;11:670-675.

[12] Ueshima H, Yoshioka A, Sumi C, et al. [Combined use of continuous epidural anesthesia nand transversus abdominis plane block for postoperative management of cesarean delivery in a patient whose previous cesarean deliveries were not properly controlled]. Masui. 2014;63:561563.

[13] Matsukawa S, Ishii H, Fukuda K. Successful anesthetic management of tracheal reconstruction and omentopexy with thoracic epidural anesthesia and transversus abdominis plane block. Masui. 2011;60:1387-1390.

How to cite this article: Tzu-Chun Wang, Chia-Hsiang Huang. Low Concentration Epidural Anesthesia Combined with Transversus Abdominis Plane Block - A Case Report in A High-risk Colostomy Operation. Signa Vitae. 2021;17(1):205-208. doi:10.22514/sv.2020.16.0061. 